\title{
INVESTIGATION OF THE ANTHOCYANIN CONTENT AND STABILITY OF CRUDE EXTRACTS FROM RED ONIONS (ALLIUM CEPA L.) GROWN IN ROMANIAN AREAS
}

\section{(c) Oancea Simona ${ }^{1}$, Draghici Olga ${ }^{1}$, Stoia Mihaela ${ }^{2}$, Sand Camelia ${ }^{1}$}

${ }^{1}$ Lucian Blaga University of Sibiu, Department of Agricultural Sciences, Food Industry and Environmental Protection, Romania 'Lucian Blaga University of Sibiu, Faculty of Medicine, Romania

Among the classes of flavonoids, anthocyanins are considered the most active compounds contributing to the high antioxidant potential. Although onion production and consumption plays a key role in Romanian agrifood system, unfortunately red onions which have higher content of phytochemicals of pharmacological interest, have not gained the same popularity as the yellow ones. The aim of this study was to investigate the anthocyanin content $(A C)$ of seven red onion cultivars from Romania and the $\mathrm{pH}$ and thermal stability of the obtained crude extracts. Several acidified and non-acidified ethanolic solutions were used in order to obtain high extraction yields in a discontinuous process. AC in dry skin of red onions, determined by $\mathrm{pH}$ differential spectrophotometrical method, was found higher than in fleshy bulb. Among investigated cultivars, the Red of Turda showed

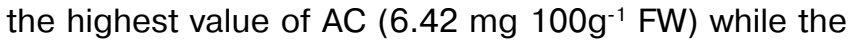
De Agnita showed the lowest AC (0.65 mg $\left.100 \mathrm{~g}^{-1} \mathrm{FW}\right)$. The results of the $\mathrm{pH}$ stability investigation of the crude extract performed during a 10-day period, showed higher stability under strong acidic conditions. Thermal analysis performed by differential scanning calorimetry (DSC) on the Red of Turda, indicated multiple events between exothermic peaks above $50{ }^{\circ} \mathrm{C}$ as result of induced chemical reactions which may influence the onion quality. At $t>100^{\circ} \mathrm{C}$ glass transitions were observed. The obtained results are important for the phytochemical characterization in terms of $\mathrm{AC}$ of red onions grown in Romanian area, in support of indigenous cultivars, being relevant for future epidemiological studies regarding the intake of anthocyanins through the diet. Although red onions are good sources of anthocyanins, the content differs greatly among cultivars. The dry skin of red onion is non-edible, but accumulates high levels of anthocyanins, showing great potential for developing natural bioactive ingredients with functional properties.

Acknowledgement: This work was supported by a grant of the Romanian National Authority for Scientific Research, CNCS - UEFISCDI, project number PN-II-ID-PCE-2011-3-0474.

\section{PHYSICAL AND CHEMICAL PROPERTIES, ANTICOAGULANT AND ANTIOXIDANT ACTIVITY OF FUCUS DRY EXTRACT}

\section{(C) Obluchinskaya E. D.}

Murmansk Marine Biological Institute of the Kola Scientific Centre of the Russian Academy of Sciences (MMBI KSC RAS), Russia

Fucus algae are natural sources of biological active substances (BAS). They contain mannitol, alginates, fucoidan, laminaran, lipids, polyphenols and others. Fucus vesiculosus is the most studied and perspective species of Fucoids because it is widespread and easily accessible for gathering and procurement. We developed and patented technology of fucus complex treatment (the Patent of the Russian Federation № 2337571); one of target products is fucus dry extract (FDE). The purpose of this research is to study physical and chemical characteristics of FDE, its anticoagulant and antioxidant activity. FDE is collected by patented method, from the Barents Sea air-dry algae F. vesiculosus. Physical and chemical characteristics were defined by modified (1) and standard (GPh XI) techniques: description, solubility, authenticity, presence of sulphatic ashes and heavy metals, content of fucoidan, polyphenols in recalculation on phloroglucinol, humidity, hygroscopic- ity. On the base of Pharmacy Institute (St.-Petersburg) research of FDE anticoagulant activity in average and double therapeutic doses at skin application and comparison preparation of heparin ointment were carried out. Prothrombin time (PT) and activated partial thrombin time (APTT) within animals were defined. Presence of statistically significant lengthening of PT and APTT (medium resorptive action) comparable to action of control sample in average and double therapeutic doses of FDE was established during experiment. The antioxidant activity of FDE was determined by using 2.2-diphenil-1-picrilhydrazine (DPPH) as a source of free radicals (2). It is established, that FDE possesses high antioxidant activity.

References: (1) Obluchinskaya E.D., Minina S.A. Pharm. Chem.J., 2004, 38 (6): 323326. (2) Brand-Williams E., Cuvelier M.E., Berset C. L.W.T., 1995, 28: 25-30. 